\title{
Numerical simulations of the charging of dust particles by contact with hot plasmas
}

\author{
W. J. Miloch ${ }^{1}$, H. L. Pécseli ${ }^{2}$, and J. Trulsen ${ }^{1}$ \\ ${ }^{1}$ University of Oslo, Institute of Theoretical Astrophysics, P.O. Box 1029 Blindern, 0315 Oslo, Norway \\ ${ }^{2}$ University of Oslo, Institute of Physics, P.O. Box 1048 Blindern, 0316 Oslo, Norway
}

Received: 21 May 2007 - Revised: 16 August 2007 - Accepted: 27 August 2007 - Published: 4 September 2007

\begin{abstract}
Charging of individual dust particles in contact with hot plasmas is studied by numerical methods. The dust particle is treated as a rigid solid body, composed by either perfectly insulating or conducting material. The collisionless plasma, consisting of electrons and singly charged ions, is simulated by Particle-in-Cell methods in two spatial dimensions. It is demonstrated that the surface conditions, i.e. roughness, of the dust particles are significant for the charging. In a streaming plasma, a dust grain develops an electric dipole moment which varies systematically with the relative plasma flow. The strength and direction of this dipole moment depends critically on the material. We observe also Langmuir oscillations excited in the vicinity of the particles, and analyze the spatial variation of their spectral distribution.
\end{abstract}

\section{Introduction}

The interactions between dust particles and warm plasmas represent a variety of often very complicated problems, where not all questions are amenable for theoretical or analytical studies, not even when individual dust particles are considered (Piel and Melzer, 2002; Fortov et al., 2005). In the present work we analyze the charging of a single dust particle by numerical methods. The analysis is carried out in two spatial dimensions with Cartesian coordinates: this restricted geometry is sufficient for illustrating the basic concepts of the problem, while on the same time allows many relevant simulations to be performed within an overseeable time. The results can be directly relevant for elongated objects (not necessarily dust particles) embedded in warm laboratory or space plasmas, for instance long wires with diameters larger than the Debye length.

Correspondence to: H. L. Pécseli

(hans.pecseli@fys.uio.no)
We find two properties of the dust particles to be important: 1) There is a pronounced distinction between perfectly conducting and insulating materials. 2) The surface conditions, i.e. the roughness of the material, influence the results noticeably. For isotropic thermal plasmas some of our results could be foreseen, at least qualitatively, by simple arguments. The situation is, however, significantly complicated when dust particles are exposed to streaming plasmas (or alternatively, the particles are moving with respect to a stationary plasma). We study the charging of dust particles with scale sizes exceeding the total Debye length, $\lambda_{D}$ (having $\lambda_{D}^{-2} \equiv \lambda_{D e}^{-2}+\lambda_{D i}^{-2}$, in terms of the electron and ion Debye lengths). Particular attention is given to the dipole moment that develops for a charged dust grain, when there is a relative velocity between the particle and the plasma. We demonstrate that this electric dipole moment exists for insulating as well as conducting particles, but its magnitude is significantly different, and also the direction is opposite for the two cases. Contrary to what might have been expected, the dipole moment is not short-circuited for a perfect conductor, but is for this case imposed by the surroundings, the wake electric field in particular.

\section{Numerical code}

The numerical analysis is carried out by a Particle-In-Cell (PIC) code. The basic features of the program are standard, and need not be discussed in any detail here (Birdsall and Langdon, 1985), so we mention just some specific features. The problem is analyzed with two boundary conditions for Poisson's equation, one at the outer boundaries, where ions and electrons are injected according to a priori chosen velocity distributions. Particles are initially also distributed internally in the simulation area (except for regions occupied by dust), according to the selected velocity distributions. Particles can leave freely across any outer boundary. The second

Published by Copernicus Publications on behalf of the European Geosciences Union and the American Geophysical Union. 


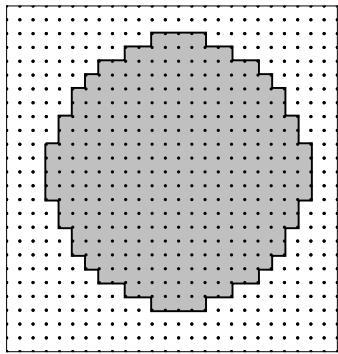

O

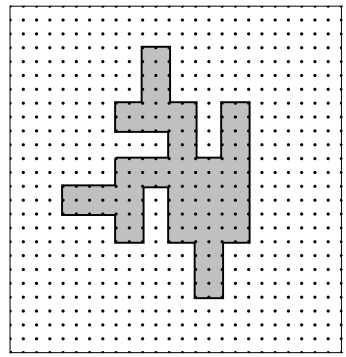

b
Fig. 1. Illustration of dust shapes considered in the present study. Shape (a) is as close to a circle as possible with the given grid resolution, while shape (b) is used for studies of very irregular surfaces.

inner boundary defines the nature of the individual dust particles. For perfect insulators, we assume that a charged particle hitting the surface remains at that position for all later times, contributing to the total electric fields. For perfect conductors, we have that the electrostatic potential is the same for all positions at the boundary, the electric field being perpendicular to the surface. The electric fields vanish inside the conductor. The dust particles are assumed to be rigid and massive, i.e. they retain their initial shape and remain immobile for the duration of the simulation. Our simulations with conducting materials have many properties common with related studies of Langmuir probe performance in collisionless plasmas (Taccogna et al., 2004; Teodoru et al., 2005). Our code has thus been verified by operating it in a "Langmuir probe mode", where a fixed potential on the inner boundary is imposed externally. We here reproduced probe characteristics for standard conditions, as well as for more complicated conditions with plane probes embedded in plasmas with ionbeams (Weber et al., 1979; Skøelv et al., 1984; Armstrong et al., 1992). The code allows many dust particles to be distributed inside the simulation area, but for the present analysis we limit the case to only one.

For the present study, the code is operated with fixed potentials at all outer boundaries. We use typically $(1-5) \times 10^{5}$ simulation particles in the following analysis. We have a grid spacing of $\lambda_{D} / 2$ to resolve also the Debye length. For the reference case we use a $200 \times 200$ grid resolution $\left(n_{g}=200\right)$, giving a simulation area of $100 \times 100 \lambda_{D}^{2}$. The time resolution is $1 / 10$ of an electron plasma period. We analyze two basically different shapes of the dust particles, one with smooth and one with an irregular surface, as illustrated in Fig. 1. The example in Fig. 1a represents a shape as close to being circular as possible with the square mesh used for computations. The example in Fig. 1b is chosen to include deep cavities as well as extrusions. The particles are assumed to be of finite size, i.e. several Debye lengths in characteristic diameter. We have studied many other shapes than those shown in Fig. 1, but these are our "reference shapes" in the following.

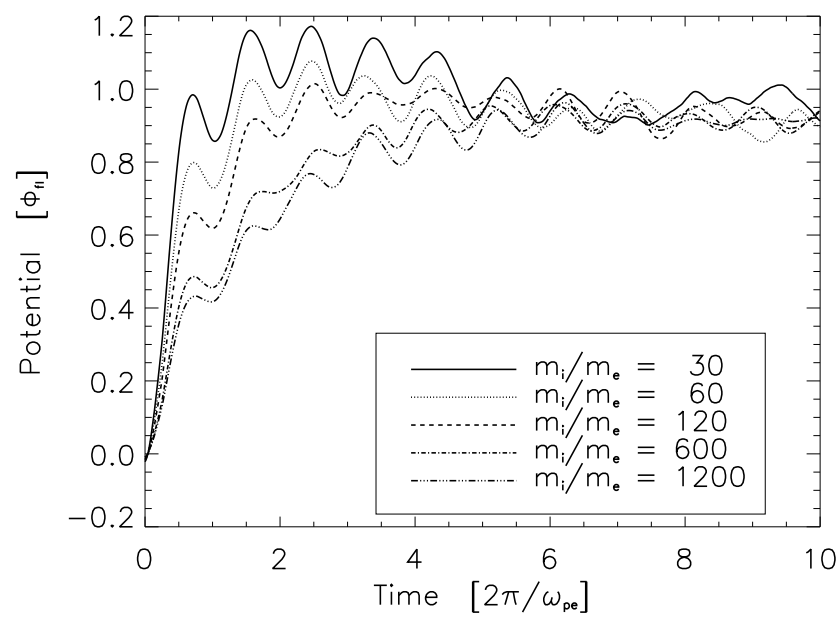

Fig. 2. Time evolution of the potential of an initially uncharged insulating dust particle, embedded at rest in a thermal plasma with $T_{e} / T_{i}=3$. Results are shown for five ion/electron-mass ratios. Times are normalized by the electron plasma period, and potentials by the floating potential (1), recalling that $\Phi_{f l}<0$.

\section{Numerical results}

We analyzed the case where the plasma was in local equilibrium with a stationary particle, and also the case with a relative velocity.

\subsection{Simulation results for thermal plasmas}

In the first part of the study, we assume that both electrons and ions have a Maxwellian velocity distribution, and that the dust particles are at rest. We solve the problem by assuming that the dust is initially uncharged. The time evolution of the absolute value of the potential for an insulating particle with the shape (a) in Fig. 1 is illustrated in Fig. 2, for different ion/electron mass ratios. The figure shows the relaxation to the floating potential, superimposed by an oscillatory component, with a frequency close to the electron plasma frequency. This latter component is associated with sheath oscillations, which are excited by the initial condition. Because the potential at $t=0$ is different from the floating potential, $\Phi_{f l}$, the initial condition acts as a step-like perturbation of the system. The initial time variation, up to approximately one half electron plasma period, is almost identical for the five cases shown. The process is subsequently slowed-down on a time scale which is scaling approximately with the square root of the mass ratio. Note that curves for increasing mass ratio lie at successively smaller ordinates in the initial temporal phase. We also note that the saturated floating potential value is reached on a time which is smaller than the ion plasma period. The initial potential of the dust particle is not exactly zero due to the random initial distribution of charges. The oscillations observed in Fig. 2 have negligible influence on 
the current to the dust particle, and after a transient period, the electric field close to the surface varies only little. For small mass ratios, $M / m \leq 60$ we note a small "overshoot" in the dust potential at the early phase of the charging. This signature becomes inconspicuous as the mass ratio is increased. We attribute this overshoot to unphysically small mass ratios ( $M / m<100)$, and use $M / m=120$ in the following. For these mass ratios the results will not be quantitatively correct, but the normalized presentation used in Fig. 2 and later figures can give also quantitatively correct results. For magnetized plasmas, the choice of mass ratio deserves particular scrutiny (Mälkki, 1994), but these arguments do not affect the present analysis of unmagnetized plasmas.

We studied the time-evolution of the dust potential for different electron temperatures, see Fig. 3. Also, we show results for different numerical grid spacings. The amplitudes of the oscillations at the plasma frequency are changing, with the largest temperature giving the smallest amplitude, but the overall averaged time-variation is basically the same. As an illustration, we also show the results obtained by simulations on a $100 \times 100$ grid $\left(n_{g}=100\right)$, which gives almost identical results. This observation indicates that the grid resolution used here is giving robust results.

Usually the time evolution of an initial value problem has little practical relevance, but as argued in Sect. 3.2 the characteristic time scale for the charging has important consequences for modeling the forces on moving charged dust particles.

We find generally that the initial transient charging time is well approximated by the electron plasma period. The subsequent relaxation to a stationary floating potential scales, for homogeneous conditions, approximately by the ion plasma period, as we might expect. The ultimate stationary potential level is given by the floating potential associated also with a Langmuir probe biased to conditions where the probe current vanishes. We use the expression for the floating potential with respect to the plasma potential for a large probe in units of $\lambda_{D}$. Taking the ion acceleration through a pre-sheath into account (the Bohm condition (Chen, 1984; Boeuf and Punset, 1999)), we have

$\Phi_{f l}=-\frac{\kappa T_{e}}{2 e}\left(\ln \left(\frac{M}{2 \pi m}\right)+1\right)$

where it is implicitly assumed that $T_{e} \gg T_{i}$. The result (1) is independent of the plasma density. The numerical figure 1 in the parenthesis in Eq. (1) originates from the presheath, which is necessary to pre-accelerate the ions. We will use Eq. (1) for normalization several times in the following, noting that for parameters relevant for our study we have $\Phi_{f l}<0$. The floating potential depends only logarithmically on the mass ratio, and it is therefore not critical to have completely realistic values of $M / m$ in the simulations. The result (Eq. 1) is inaccurate for realistic finite ion temperature conditions by implicitly assuming $T_{i} \approx 0 \mathrm{~K}$. The time asymp-

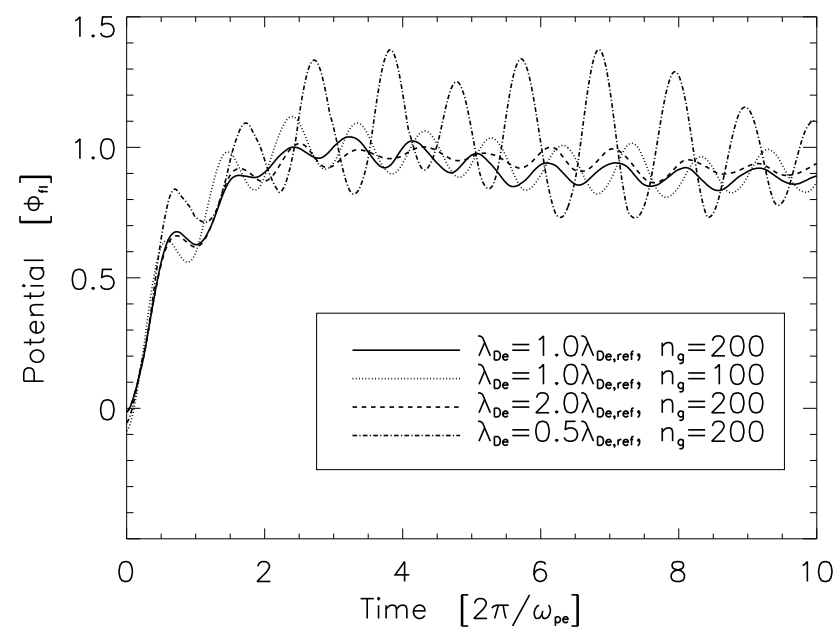

Fig. 3. Time evolution of the potential of an initially uncharged insulating dust particle, embedded at rest in a thermal plasma. Results are shown for three electron temperatures, measured in relation to the reference temperature in Fig. 2. Times are also here normalized by the electron plasma period, and potentials by the floating potential (1), which is here changing with the electron temperature. Full line shows the reference case from Fig. 2. We also have a curve for the case $n_{g}=100$, where the grid resolution is reduced by a factor $1 / 2$, as compared to the reference case. We have $M / m=120$.

totic potential in Fig. 2 is nonetheless well approximated by the standard analytical estimate Eq. (1).

The result (Eq. 1) refers to the case where the dust grain has a diameter greater than the Debye length, $\lambda_{D}$. For the opposite limit, we can determine an analytical expression for the floating potential by orbit theory, for instance (LochteHoltgreven, 1968).

For smooth surfaces as in Fig. 1a, the potential variation around the dust grain shows the usual Debye shielded potential. For irregular surfaces we can have an irregular potential distribution around the dust particles, as illustrated in Fig. 4, but the irregularity of the sheath diminishes as the distance to the object increases. The properties of the local irregular electric field variation at the surface will be particularly important when considering the coagulation of two dust grains.

For the present conditions, we find the nonlinear corrections to the linear Debye-shielding to be immaterial, but note that for surfaces that can be considered locally plane (i.e. when the radius of curvature is much larger than $\lambda_{D}$ ), this case can be solved analytically (Sivukhin, 1966).

\subsection{Simulation results for drifting plasmas}

As a simple generalization of the results from Sect. 3.1 we now assume that the plasma is drifting with some velocity $U$, or alternatively that the dust particle is moving with constant velocity through a thermal plasma (Lapenta, 1999; 

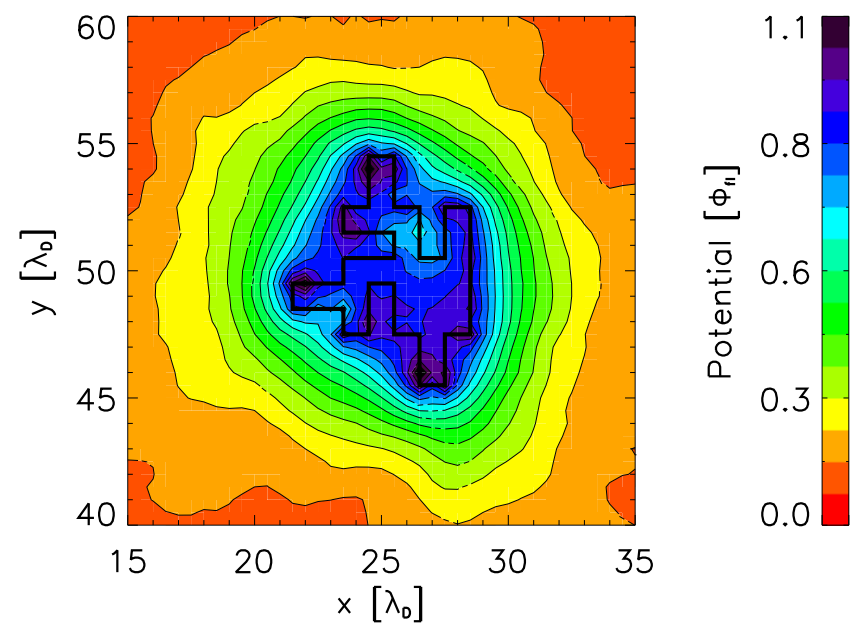

Fig. 4. Debye shielding of a dust grain of irregular shape made of insulating material as in Fig. 1b. The figure shows a section of a larger simulation area. Distances are normalized by $\lambda_{D}$, and potentials by (1), recalling that $\Phi_{f l}<0$.

Kwok et al., 2003). In particular, for supersonic particles, we expect the formation of a $V$-shaped Mach-cone behind the dust grain, where for the present conditions the finite size of the particle is likely to be of importance, and the radiation pattern will differ from that of a point charge studied elsewhere (Huld et al., 1990; Guio and Pécseli, 2003). For distances much larger than the diameter of the dust grain, we can however expect the analytical results of e.g. Guio and Pécseli (2003) to apply. We emphasize that the wake and the Machcone are entirely unrelated physical phenomena: the Machcone will be observed also for a supersonic point charge, in which case no wake is formed. We define the wake as the region in the shadow of the object, where the electron and ion densities are significantly reduced (by more than $\sim 50 \%$ ). The Mach-cone is a wave-like disturbance, emitted from a supersonic source, while the wake is caused by the finite size of the object, depending also on the distributions of the particles absorbed on the surface.

We find a significantly increased charging time, as compared to the case where the dust-grain is at rest with respect to the plasma, with representative cases illustrated in Fig. 5. The initial phase, with duration of a few electron plasma periods, is almost indistinguishable from the results in Fig. 2, while the subsequent time evolution of the net charge is considerable slower. For large flow velocities, the potential becomes also increasingly fluctuating, and the estimate on, for instance, the charge on the dust particles becomes somewhat more uncertain. Dust particles moving at high speeds through inhomogeneous plasmas will at any given position have a charge that depends on the prior orbit, in general. We should like to mention that the characteristic charging-time $T_{C}$ is an important parameter for particles propagating in an

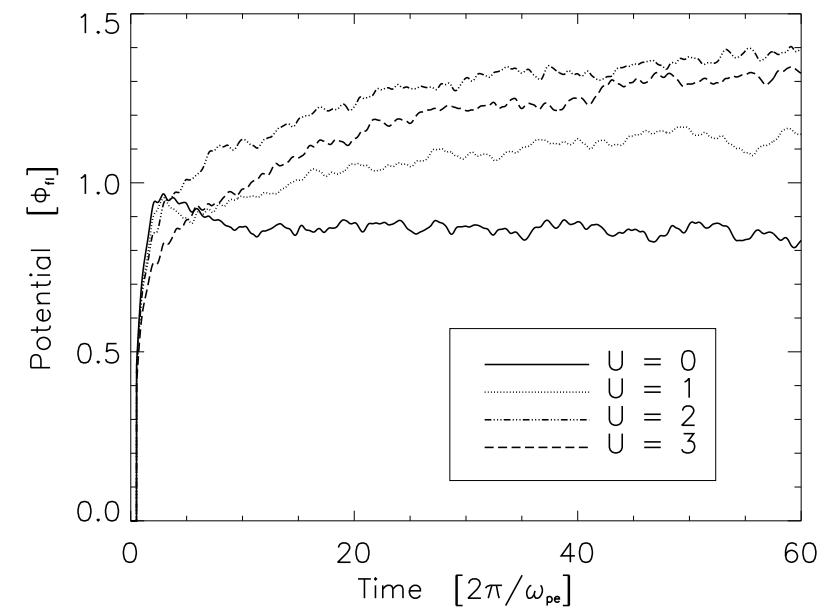

Fig. 5. Time evolution of the potential of an initially uncharged insulating dust particle, embedded in a flowing plasma with $T_{e} / T_{i}=3$. We have $M / m=120$. Times are normalized by the electron plasma period, and potentials by the floating potential (1). We show four cases: $U=0,1,2,3$ in units of $C_{S}$. The oscillations at the electron plasma frequency have been removed by filtering to improve the presentation.

inhomogeneous plasma: given the particle velocity $U$, we can define a time scale associated with the inhomogeneity as $\tau_{\text {in }} \equiv \mathcal{L} / U$, where $\mathcal{L}$ is a characteristic scale-length of the plasma inhomogeneity. If $T_{c}<\tau_{i n}$ we can argue that the dust charge is at all times derived from local equilibrium conditions in the plasma, while in the opposite case, $T_{c}>\tau_{\text {in }}$, there is a "memory" in the system, and the actual charged state of the dust will depend on the preceeding motion through the plasma. Assuming that the dust is moving in a stationary electrostatic field, the forces on a dust grain are generally not conservative when the charged state of a dust grain depends on its path (Raadu and Shafiq, 2003; Shafiq and Raadu, 2004; Zhdanov et al., 2005).

The most conspicuous difference in the electrostatic fields around a dust grain at rest and one moving with respect to the plasma is the asymmetry developing due to the shadow or wake behind the particle. In many ways these features are similar for microscopic dust grains and macroscopic objects like space-crafts (Singh et al., 1986, 1989) or the moon (Nakagawa and Iizima, 2005). We will here consider only relative velocities well below the electron thermal speed, and may safely assume that the object is exposed to an almost isotropic and uniform electron flux. The ion flux is, on the other hand, anisotropic, in general. The part of the dust grain facing the flow direction experiences the largest ion flux. Consequently an electric field builds up inside the dust particle for insulating materials. This electric field may become so large that the particle is disrupted. (If we consider small organisms like bacteria as equivalent to dust grains, these features may actually be used for sterilization (Shukla 

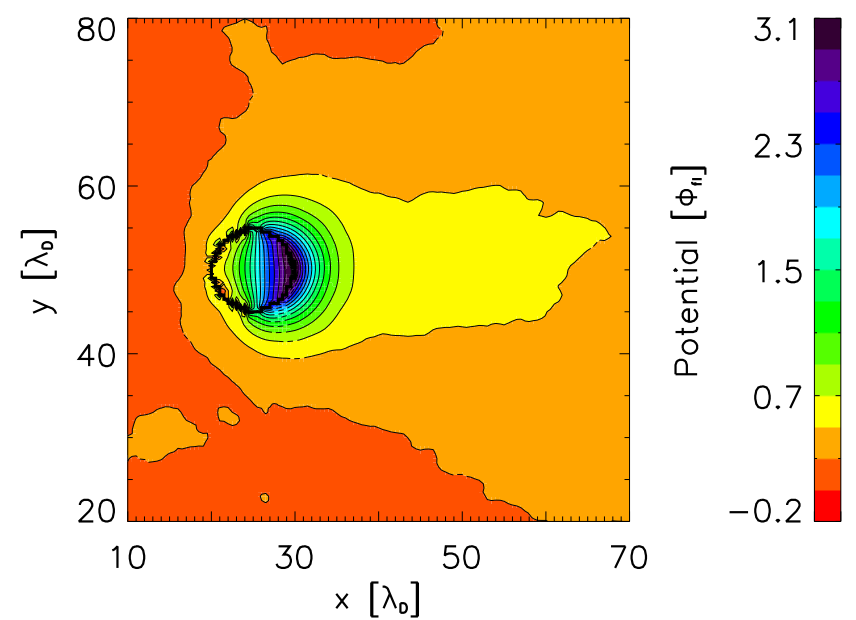

Fig. 6. Time asymptotic potential distribution (color coded) around an insulating dust grain in a flowing plasma. The particle shape is given by Fig. 1a. We have $U=2 C_{s}$. Note the large potential variation inside the dust grain. Also here we normalize distances by $\lambda_{D}$, and potentials by $\Phi_{f l}$ from Eq. (1).

and Mamun, 2002; Laroussi et al., 2003).) For a perfectly conducting material, on the other hand, the surface charges have to be redistributed so as to make the internal electric fields vanishing. Consequently, we expect to find significant differences between the potential distributions around insulators and conductors when they are moving through the plasma.

In Figs. 6 and 7 we show the saturated time-stationary potential variation around a dust grain embedded in a streaming plasma (Manweiler et al., 2000), where the flow velocity is $2 C_{s}$, the temperature ratio $T_{e} / T_{i}=3$, and the ion-electron mass ratio is $M / m=120$. In normalized units, the results can be directly relevant, for instance, also for $Q$-machine conditions (Motley, 1975). When the diameter of the dust grain exceeds the Debye length $\lambda_{D}$, we can obtain an analytical estimate for the electric field inside a dust grain of insulating material by considering a small surface element locally as equivalent to a plane Langmuir probe. For a surface element at the side of the probe, the flow velocity is tangential, and the floating potential associated with that element is given as for a plasma at rest (Eq. 1). For surface elements facing towards or away from the plasma flow, we can still assume the potential as given by the floating potential of a Langmuir probe, but have to take the plasma flow into account. The foregoing estimates will be incorrect for conducting materials, where charges are redistributed to maintain a vanishing internal electric field.

For purely ballistic or free streaming ions, we can readily estimate the shape of the plasma density wake to be approximately triangular, with a length $\sim \frac{1}{2} L U / u_{t h}$, where $u_{t h}$ is a thermal ion velocity and $L$ is the diameter of the obsta-
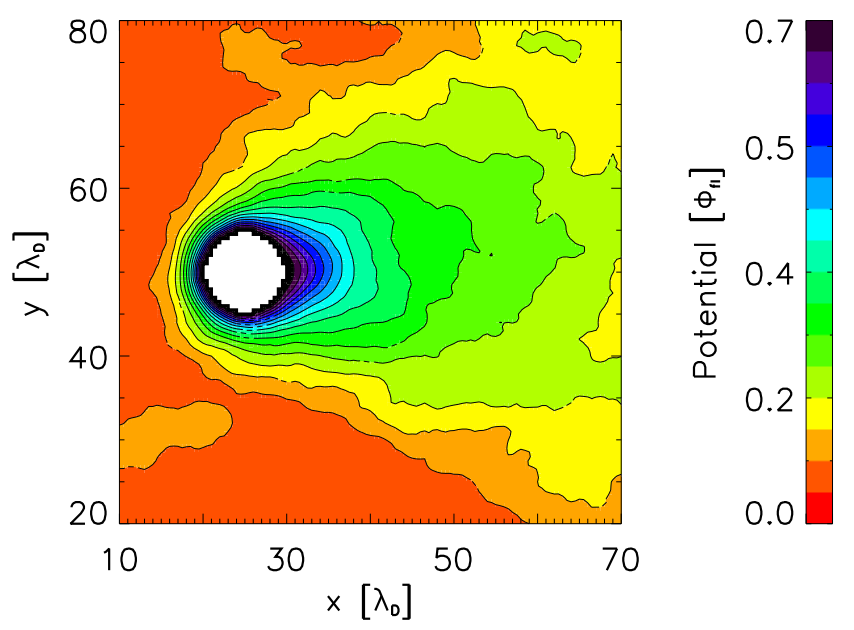

Fig. 7. The time asymptotic potential distribution (color coded) around a dust grain made of a perfectly conducting material embedded in a flowing plasma. The shape of the particle is given by Fig. 1a. The potential is constant inside the dust grain in this case, and a white color is used there for improving the presentation. We have $U=2 C_{S}$.

cle in the flow, measured in the direction perpendicular to the flow vector. More accurately, but still with free streaming ions having a Maxwellian distribution, we can determine the density variation in a two-dimensional wake by assuming quasi-neutrality. These estimates ignore the ion acceleration by the ambipolar electric fields. The ions are accelerated, giving a triangular shape of the wake, with an approximate length $\frac{1}{2} L U / C_{s}$. This latter estimate gives a value which is too low when the flow velocity is sub-sonic. If we have approximate thermal equilibrium, $T_{e} \approx T_{i}$, the ambipolar electric fields have little importance, and the free streaming wake can be used as a reasonably accurate estimate for sub-sonic flows. For sub-sonic flows with $T_{e}>T_{i}$, but with velocities exceeding the ion thermal velocity $u_{t h}$, we expect distorted, and non-triangular, shapes for the wake as observed for instance in numerical simulations (Guio and Pécseli, 2005).

The plasma streaming creates an ion wake behind the dust grain. The electron pressure forces electrons into this wake, and the resulting charge imbalance creates an ambipolar electric field which accelerates ions into the wake as well. For the insulating material, the charges are negative on the surface facing away from the flow, and these charges subsequently attract the ions. For conducting materials, the surface charges on the back side have to be compensated by the charge redistribution in order to cancel the internal electric fields, and the ion dynamics in the wake will be altered substantially as compared to the insulating case. By comparing Figs. 6 and 7 we find that the range of variation of the electrostatic potential is much smaller for the conductor, as compared to the insulating material, the difference being caused by the 


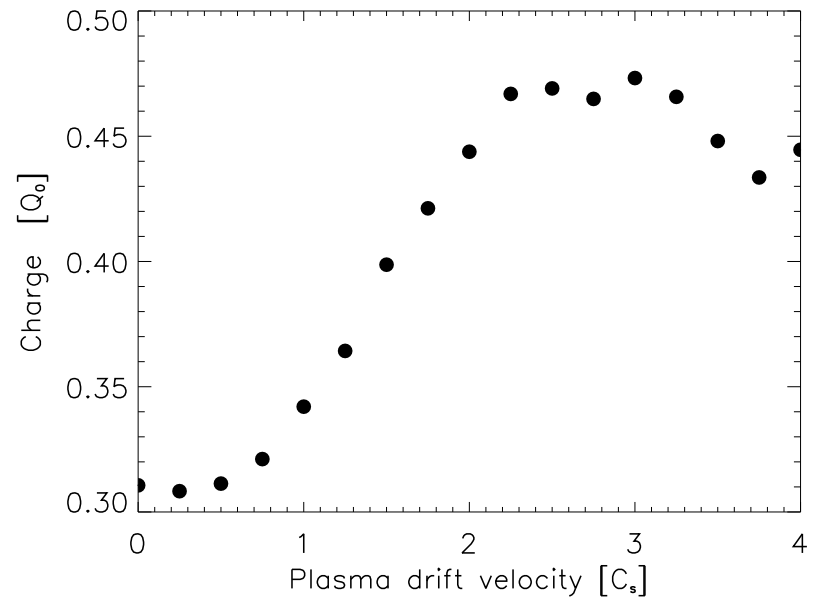

Fig. 8. Absolute value of the normalized average charge on an insulating dust grain with shape as in Fig. 1a, for different plasma flow velocities. Velocities are normalized by the sound speed $C_{S}$ and charges by $Q_{0}$ from Eq. (2), recalling that $Q_{0}<0$.

charge redistribution just mentioned. We note the presence of a Mach-cone, best seen in Fig. 7. For the present case, with $U=2 C_{s}$, we have the opening angle of the cone to be $\theta=30^{\circ}$, which is close to the observed value within the observational accuracy. We should note that the sound speed was here deduced from the electron and ion temperatures of the undisturbed plasma, so a perfect agreement can not be expected since the particle distribution functions are significantly disturbed in the vicinity of the dust grain. We obtain the sound speed by $C_{s}=\sqrt{\kappa\left(T_{e}+\gamma T_{i}\right) / M}$, with $\gamma=5 / 3$ for adiabatic ion dynamics. We also note that the Mach cone pattern is significantly damped with distance away from the particle, and does not extend to more than $\sim 50 \lambda_{D}$, consistent with analytical results (Guio and Pécseli, 2003), where the effects of the kinetic Landau-damping are included. The diagnostic potential in the observations of Mach cones (which are observable from large distances) has been noted (Havnes et al., 1996).

The normalized charge accumulated on a dust particle for different flow velocities is shown in Figs. 8 and 9. The shape of the particle is in both cases given in Fig. 1a. We obtain a normalizing charge $Q_{0}$ per length unit $\ell$ in the direction perpendicular to the simulation plane by considering the isotropic case without flow. The physical dimension of $Q_{0}$ is Coulomb/length. We argue that for cylindrical symmetry the electrostatic shielded potential is given by $a K_{0}\left(r / \lambda_{D}\right)$, where by Eq. (1) we have $a=\Phi_{f l} / K_{0}\left(R / \lambda_{D}\right)$, with $K_{0}$ being the modified Bessel function and $R$ the radius of the dust grain. The radial electric field is then $E_{r}=\left(a / \lambda_{D}\right) K_{1}\left(r / \lambda_{D}\right)$. For a conducting material, as well as for insulators with cylindrical symmetry, the electric field inside the dust grain vanishes when the charge is restricted to the surface. The electric field then also determines the surface charge density $\sigma=\varepsilon_{0} E$. We thus obtain $Q_{0}=2 \pi R \varepsilon_{0}\left(a / \lambda_{D}\right) K_{1}\left(R / \lambda_{D}\right)$, or

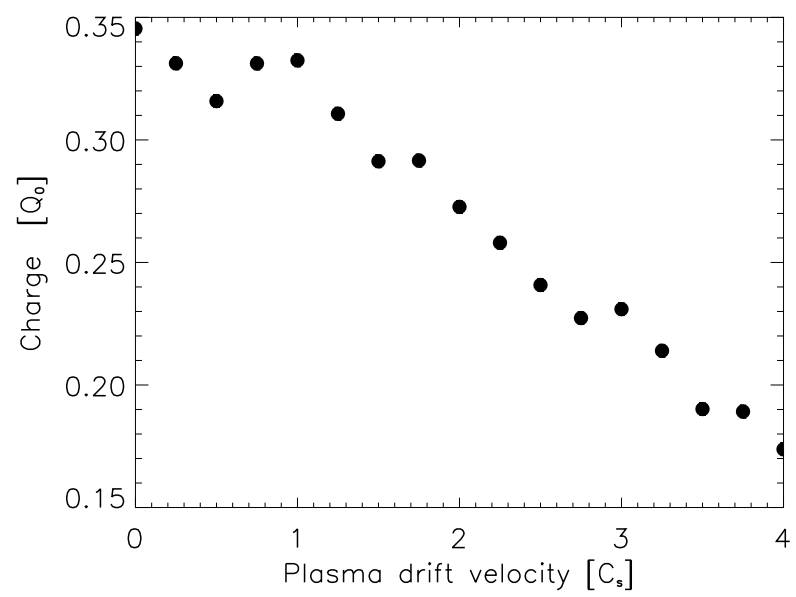

Fig. 9. The normalized average charge on a conducting dust grain with shape as in Fig. 1a, for different plasma flow velocities. Velocities are normalized by the sound speed, $C_{s}$, and charges by $Q_{0}$ from Eq. (2), recalling that $Q_{0}<0$.

$Q_{0}=-\pi \varepsilon_{0} \frac{\kappa T_{e}}{e}\left(\ln \left(\frac{M}{2 \pi m}\right)+1\right) \frac{R}{\lambda_{D}} \frac{K_{1}\left(R / \lambda_{D}\right)}{K_{0}\left(R / \lambda_{D}\right)}$.

We will use $Q_{0}$ for normalizations of relevant quantities in the following, noting that $Q_{0}<0$ for these cases. The reference charge is a measurable quantity (Homann et al., 1999), and has general interest for the dust dynamics, but as stated, the present derivation refers to stationary dust grains only. The interesting problem is how this charge varies with velocity, as summarized here by Figs. 8 and 9. While net charges can be determined experimentally, the distribution of charges on the surfaces can be found only by numerical simulations.

The number of elementary charges on the dust grain is here approximately given by

$$
\frac{Q_{0} \ell}{e} \approx \pi \ell \lambda_{D}^{2} n_{0}\left(\ln \left(\frac{M}{2 \pi m}\right)+1\right) \frac{R}{\lambda_{D}} \frac{K_{1}\left(R / \lambda_{D}\right)}{K_{0}\left(R / \lambda_{D}\right)},
$$

which has to be truncated to the nearest integer number. The parenthesis will typically be of the order 5, so for an order of magnitude estimate we can use

$$
\frac{Q_{0} \ell}{e} \sim \ell \lambda_{D} R n_{0}=N_{p} \frac{\ell R}{\lambda_{D}^{2}},
$$

in terms of the plasma parameter $N_{p} \equiv n_{0} \lambda_{D}^{3}$, noting that the ratio of the two Bessel functions is $1<K_{1} / K_{0}<2$ for the argument $R / \lambda_{D}>0.4$. Recall that by using Eq. (1), we have implicitly assumed that the dust grain can be locally approximated by a plane surface. Consequently, the estimate (2) depends only linearly on $R$, to a good approximation. We have $N_{p} \gg 1$ for most plasmas of interest here. Assuming that the number of charges on a dust grain has a Poisson distribution, to a reasonable approximation, with $Q_{0}$ representing the average value, we will have the standard deviation of 
the charges to be $\sim \sqrt{Q_{0}}$. This implies that the relative magnitude of charge fluctuations is $\sim 1 / \sqrt{Q_{0}}$, which is a small quantity (Matsoukas and Russell, 1995). We can usually ignore the statistical variations in the number of elementary charges on dust grains when their scale sizes are larger than the Debye length.

The results summarized in Figs. 8 and 9 show that the net charge on a dust particle is reasonably close to the estimate (2) for vanishing or small plasma flow velocities. (Recall that $Q_{0}$ in Eq. (2) was derived for stationary and isotropic conditions, and enters here solely for normalizing purposes.) As the flow velocity increases, we find an increase in the net charge for the insulating case. The increase is not dramatic, though, since we need a strongly supersonic flow in order to obtain approximately a doubling of the charge. We note a maximum charge value for Mach numbers close to $\mathcal{M}=2.5$. For this and larger Mach numbers, our calculations of the net charges become increasingly more uncertain. A charge maximum for large Mach numbers of the plasma flow have been argued on theoretical grounds (Fortov et al., 2005). Note here that the largest velocity shown in Fig. 5 corresponds to a region after the charge maximum in Fig. 8. For conductors, we do not find any charge maximum, at least within the parameter regime studied here. For spherical dust particles studied in full three dimensions, somewhat different results are expected for the related problem (Fortov et al., 2005; Hutchinson, 2005). The initial trend of the charge variations with flow velocity differs for insulators and conductors. This is also observed in the spherical case (Lapenta, 1999). Significant differences, as compared to the present cylindrical case, are manifested only for large plasma drift velocities.

The potential distribution around an irregular dust particle (with shape given in Fig. 1b), is shown in Fig. 10. We note the large variation in surface charge density. Only very little charge penetrates into the bottom of the cavities, except in the cases where these cavities face directly towards the plasma flow. In a way, these results agree with those obtained for the micro-porous ion energy analyzers used in some double plasma devices. Also in these cases only those ions having a velocity vector exactly aligned with the pores of the analyzer reach the collector (Stenzel et al., 1982). We also note the large potential variations originating from the differences in charge density on the two sides of extrusions facing towards and away the plasma flow, respectively. The front part is exposed to the full plasma flow, while the back side is predominantly bombarded by the electron component. For moderate Mach numbers for the plasma drift velocity, we can assume that the electron flux to the probe-surface remains isotropic to a good approximation. Including a drift velocity into Eq. (1), we can assume that the pre-sheath is unaffected by a drift. This is probably acceptable as long as the drift velocity is much smaller than the electron thermal velocity. In that case, the ions can be assumed to arrive at the sheath edge with a velocity approximately given as $C_{s}+U$.

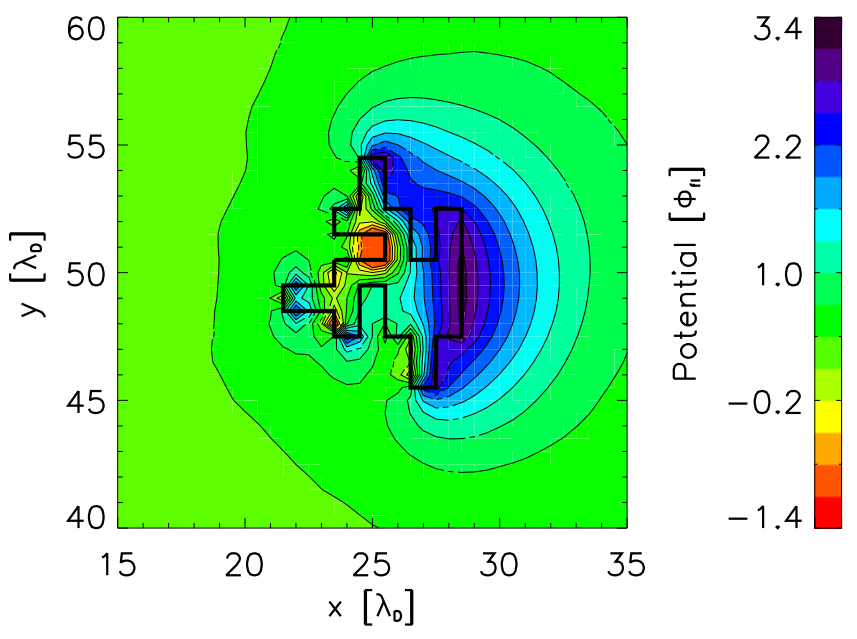

Fig. 10. Potential distribution (color coded) around a dust grain made of a perfectly insulating material embedded in a flowing plasma. The shape is given by Fig. $1 \mathrm{~b}$.

This generalizes Eq. (1) to give the approximate expression

$\Phi_{f l}=-\frac{\kappa T_{e}}{2 e}\left(\ln \left(\frac{M}{2 \pi m(1+\mathcal{M})^{2}}\right)+1\right)$,

with $\mathcal{M} \equiv U / C_{s}$ being the Mach number. The result (Eq. 3) applies for a surface facing the plasma flow. The opposite side is not exposed to any drift, and here ions enter with a velocity essentially determined by the acceleration in the presheath. At least for moderate drift velocities, $\mathcal{M} \ll 1$, we can consequently estimate the maximum potential drop across a dust grain made of insulating material by

$|\Delta \Phi|=\frac{\kappa T_{e}}{e} \ln (1+\mathcal{M}) \approx \frac{\kappa T_{e}}{e} \mathcal{M}$,

where the latter approximation applies for small Mach numbers, $\mathcal{M} \ll 1$. The expressions (3) and (4) are seemingly independent of the plasma density. We should emphasize, however, that $n_{0}$ enters implicitly by the assumption that $R>\lambda_{D}$. The estimate (4) is independent of the electron-ion mass ratio.

In Fig. 11 we illustrate the potential on selected positions on an insulating dust particle for varying plasma drift velocities. Thus, the symbol o shows the potential on the front side, which is facing the flow, while $\Delta$ shows the potential on the shadow side. With $\bullet$ we show the maximum potential difference across the particle, here with shape as given in Fig. 1a. For clarity we show the potential difference negative: the corresponding electric field points in the plasma flow direction, see also Fig. 6. For significantly super-ion thermal flow velocities (which need not be supersonic if $T_{e} \gg T_{i}$ ), the ions can not reach the shadow side of the macroscopic particle by ballistic motion, and the electron current is dominating. The electron flux to the shadow-side surface builds up an electric 


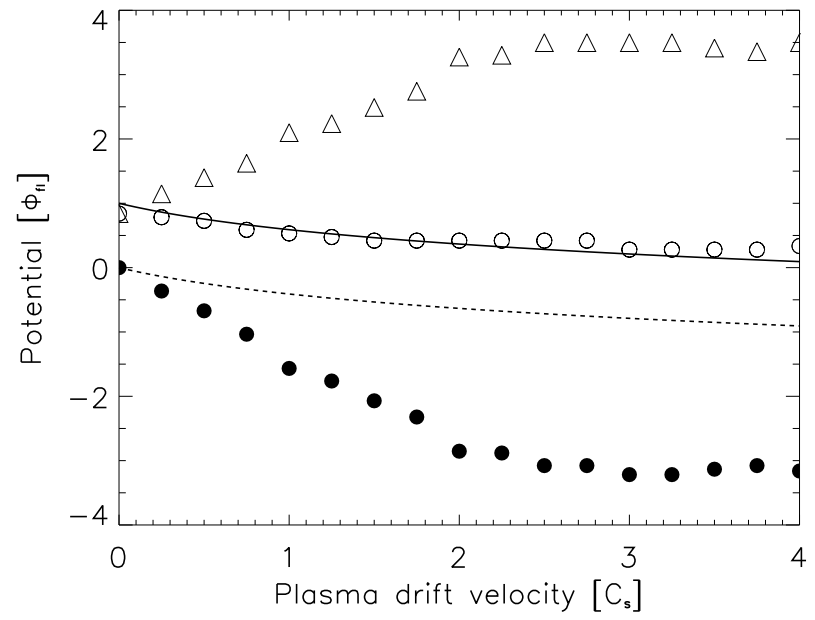

Fig. 11. Front and back potentials on an insulating dust particle for varying plasma drift velocities. The symbol $\circ$ shows the potential on the front side, facing the flow, while $\Delta$ is on the shadow side. With - we show the maximum potential difference across the particle, here with shape as given in Fig. 1a. Full and dashed lines give the analytical functions (3) and (4), respectively. The normalizing potential $\Phi_{f l}$ is given by Eq. (1).

field, until it is strong enough to accelerate passing ions into the wake.

For plasma flow velocities $C_{s}<U \ll u_{t h e}$, one possibility could be that the shadow side of the particle, facing directly opposite the plasma flow vector $\mathbf{U}$, would be bombarded solely by the electron population. In that case we would expect a potential given approximately as $\kappa T_{e} / e$, i.e. a constant quantity given by $\Phi_{f l}$ in Eq. (1) apart from a numerical constant and independent of the plasma flow velocity. Inspection of Fig. 11 shows that the shadow-side potential varies with the plasma flow velocity, and we can therefore argue that energetic ions are deflected to reach into the wake.

Full and dashed lines in Fig. 11 give the analytical functions (3) and (4). Note that the potential difference $|\Delta \Phi|$ reaches saturation at the same flow velocity where the charge on the dust particle reaches the maximum level, see Fig. 8. We find that the potential on the front side is reasonable well accounted for by Eq. (3), qualitatively as well as quantitatively, while the shadow side potential varies quite differently, as soon as the flow velocity exceeds a small fraction of the sound speed. Consequently, also the analytical result (4) will have a limited range of validity, $\mathcal{M}<0.2$.

Considering again bacteria as equivalent to dust particles, we may estimate the plasma flow velocity necessary to build up an electric field sufficient for their disruption (Shukla and Mamun, 2002; Laroussi et al., 2003). We take as an example a plasma with $T_{e} \approx 0.5 \mathrm{eV}$, which is representative for many discharge plasmas. A typical scale size of the bacteria can be $R_{b} \approx 10 \mu \mathrm{m}$, and the stress necessary to break the organism (the tensile strength, expressed as the force per unit area) is estimated to be $F_{t} \approx(1-5) \times 10^{6} \mathrm{dyn} \mathrm{cm}^{-2}=(1-5) \times 10^{5} \mathrm{~Pa}$. For the given value of $T_{e}$, we need a plasma density $n_{0}>3 \times 10^{12} \mathrm{~m}^{-3}$ to give $R_{b}>\lambda_{D}$. The force on the surface charges that produce the internal electric field can be estimated as $F \approx \sigma E$, where $\sigma$ is the surface charge density. As an estimate for the electric field inside and close to the surface of an insulator we can use $E \approx \frac{1}{2} \sigma / \varepsilon_{0}$, giving $F \approx \frac{1}{2} \varepsilon_{0} E^{2}$ i.e. the electric field energy density, which for electrostatic conditions can as well be expressed as $F \approx \frac{1}{2} \varepsilon_{0}(\nabla \phi)^{2} \sim \frac{1}{2} \varepsilon_{0} \phi^{2} / R_{b}^{2}$. To have $F \approx F_{t}$ it turns out that we require $\mathcal{M} \gg 1$, which is hardly feasible, and the range of validity of Eq. (3) is exceeded anyhow. Referring to Fig. 4 for insulating materials, we note, however, that even in the absence of any plasma flow, we can have large electric fields at extrusions from any object, bacteria in particular (Shukla and Mamun, 2002). These extrusions can be seriously damaged even in the absence of any plasma flow (Laroussi et al., 2003). Plasma flows thus have little importance for sterilization, in this context.

The strong anisotropy of the ion flux to the surface of the dust particle in a streaming plasma gives rise to a dipole moment of the resulting charge distribution (Manweiler et al., 2000; Ivlev et al., 1999). Previous related works (Lapenta, $1998,1999)$ studied a particle of radius $R=0.1 \lambda_{D e}$ in cylindrical coordinates in a plasma with drifting ions by using PIC-simulations. The ion to electron mass ratio was there $M / m=100$, and the temperature ratio $T_{e} / T_{i}=5$. It was found there that the electric dipole moment develops only on dielectric particles, while the conducting particles were assumed to charge homogeneously, and that the electric field around the conducting particle is symmetric. In the present study, see Figs. 12a and b, we find that 25 times larger conducting particles (particle radius $R \approx 2.5 \lambda_{D e}$ ) acquire a significant electric dipole moment in plasmas with the ion drift velocities larger than the ion thermal velocity, and that the ion drift leads to a strong anisotropy in the potential distribution in space. This electric dipole moment is, however, one order of magnitude lower than for corresponding insulating dust particles. In either case we find a saturation of the dipole moment for large plasma flow velocities, $U / C_{s}>2$. The plasma flow velocity is normalized by the sound speed, while the electric dipole moment is normalized by $Q_{0} D$, again with $Q_{0}$ given by Eq. (2), while $D$ is the maximum length of the dust grain along the direction of the plasma flow. The two quantities $Q_{0}$ and $D$ appear natural choices for the normalizations, but we emphasize that there are no reasons to expect dipole moments having precisely the value $Q_{0} D$.

\section{Langmuir oscillations}

Langmuir waves are excited in the system. These oscillations are weakly damped in the case without plasma flow, while they seem to prevail during the simulations for the case where 


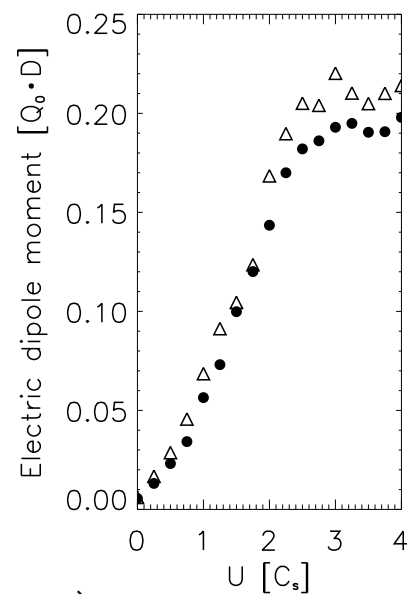

a)

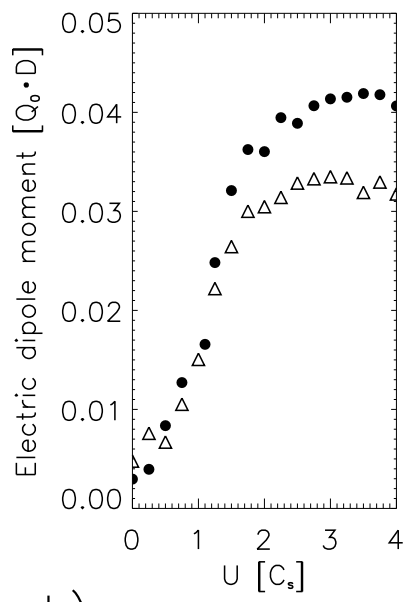

b)

Fig. 12. The absolute value of the dipole moment of a dust particle embedded in a streaming plasma, shown as a function of drift velocity (in units of $C_{S}$ ). In (a) we show the case of insulating material, in (b) a perfect conductor. The shape of the dust grain as given in Fig. 1a is illustrated by $\bullet$, while the shape given in Fig. $1 \mathrm{~b}$ is shown by $\triangle$. Ideally, we should have the dipole moment vanishing for $U=0$ : the actual value gives the uncertainty on our estimate for the dipole moment.

we have a net plasma flow past the obstacles. We will quantify the nature of these oscillations, demonstrating in particular also here a significant difference between the conducting and insulating materials.

In Fig. 13 we show for reference the variation of the electron density in the vicinity of an insulating particle. We have taken the average over many time-steps in order to reduce the noise level in the figure. The spectra for the observed Langmuir oscillations are shown in Fig. 14 for seven different spatial positions shown by dots in Fig. 13. The spectra are obtained by analyzing the full time series and then averaging over 5 neighboring frequency samples. Hereby we obtain an improvement of the spectral estimate, on the expense of frequency resolution. The same improvement can be obtained by reducing the original time series to 5 shorter ones and then take the average of the spectra (Bendat and Piersol, 1986). We find that at a position inside the sheath (the uppermost curve in Fig. 14) we have oscillations for a relatively broad frequency interval, up to the electron plasma frequency corresponding to the unperturbed density at large distances from the probe. Also outside the sheath we find oscillations at frequencies corresponding to plasma densities inside the sheath. These can not originate from waves generated within the sheath, since these can not propagate into the over-dense plasma. However, as already demonstrated by Barston (1964) for a cold plasma model, the frequency spectrum of Langmuir waves for an inhomogeneous plasma will in general at any position have a broad spectrum including all

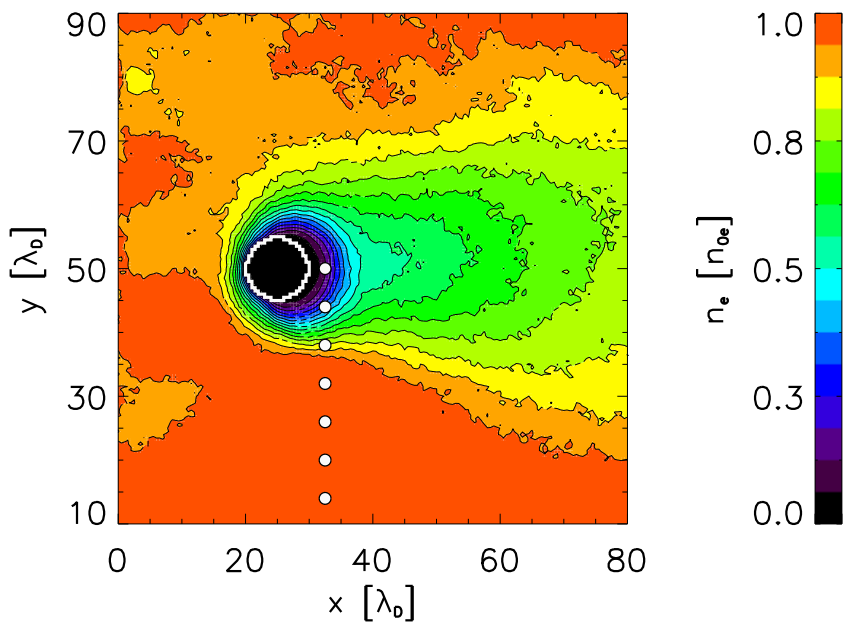

Fig. 13. Variation of the electron density in the vicinity of an insulating particle. We have $T_{e} / T_{i}=5$. A white line indicates the surface of the obstacle. We have $U=2 C_{s}$. Densities are normalized by $n_{0 e}$, which is the unperturbed ion density at large distances from the dust-grain.

plasma frequencies in the plasma, because of the non-local spatial variation of the potential resulting from an excitation at any position.

In Fig. 15 we show for reference the variation of the electron density in the vicinity of a conducting particle. The spectra for the observed Langmuir oscillations are shown in Fig. 16 for seven different spatial positions shown by dots in Fig. 15. We here note a pronounced reduction in fluctuation amplitudes within the sheath, as compared to the case of an insulating object, as in Fig. 14. Evidently, the conducting surface acts as a short circuit for the potential, which is physically reasonable for an un-symmetric sheath, as in the present case, see Figs. 13 and 15. For a completely cylindrical sheath we could in principle have the lowest order, $m=0$, mode unaffected by the composition of the object. At larger distances from the particle, the Langmuir spectra become very similar in Figs. 14 and 16. In particular we also have oscillations with $\omega>\omega_{p e}$, corresponding to the finite temperature part of the dispersion relation.

For low frequencies, below the ion plasma frequency, we find an enhanced fluctuation level, but the time series are too short to allow a detailed resolution of this part.

Langmuir oscillations are often observed to be continuously excited in space plasmas (Eriksson and Boström, 1995). Although the bandwidths are usually relatively large, the fluctuations can be used to give a crude calibration of independent plasma density measurements, by Langmuir probes, for instance. Our results indicate that a probe will usually detect a frequency somewhat below that of the plasma frequency of the surrounding plasma. 


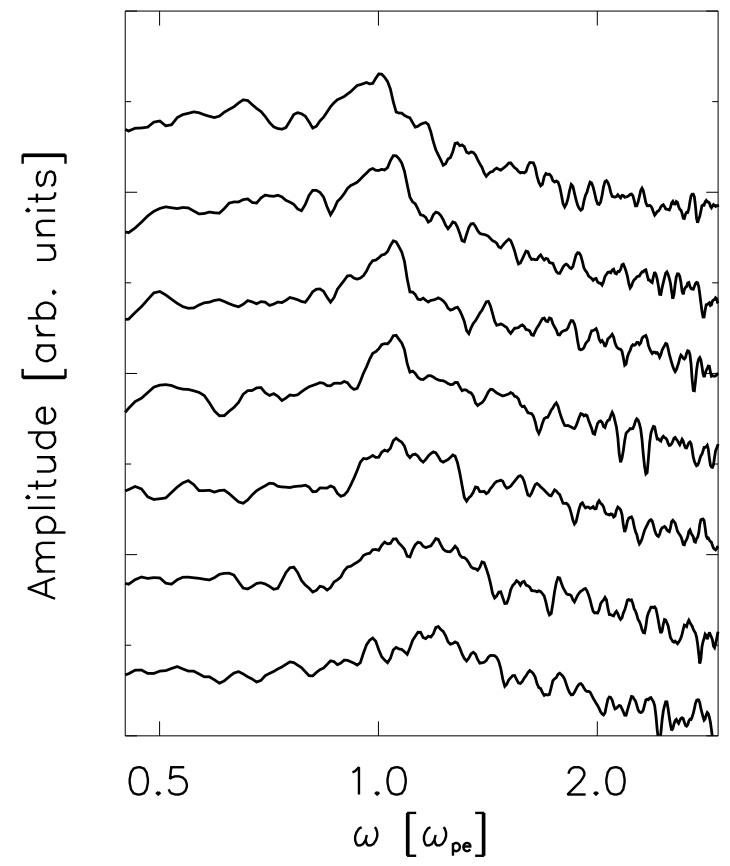

Fig. 14. High frequency spectrum of the oscillations in double logarithmic presentation at selected positions shown in Fig. 13 by open dots. Frequencies are normalized by the plasma frequency of the plasma at large distances from the particle. Tick marks on the vertical axis are with a factor of 10 separation and the individual power spectra are displaced similarly in the vertical direction.

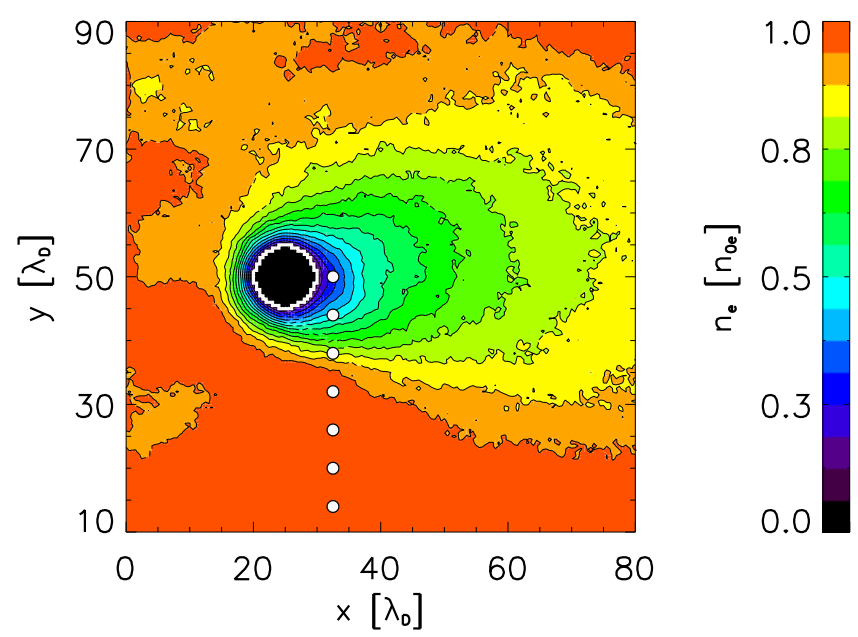

Fig. 15. Variation of the electron density in the vicinity of a conducting particle. We have $T_{e} / T_{i}=5$ and $U=2 C_{s}$. Densities are normalized as in Fig. 14.

\section{Conclusions}

The interactions between dust particles and warm plasmas have been analyzed by numerical methods. For a particle at

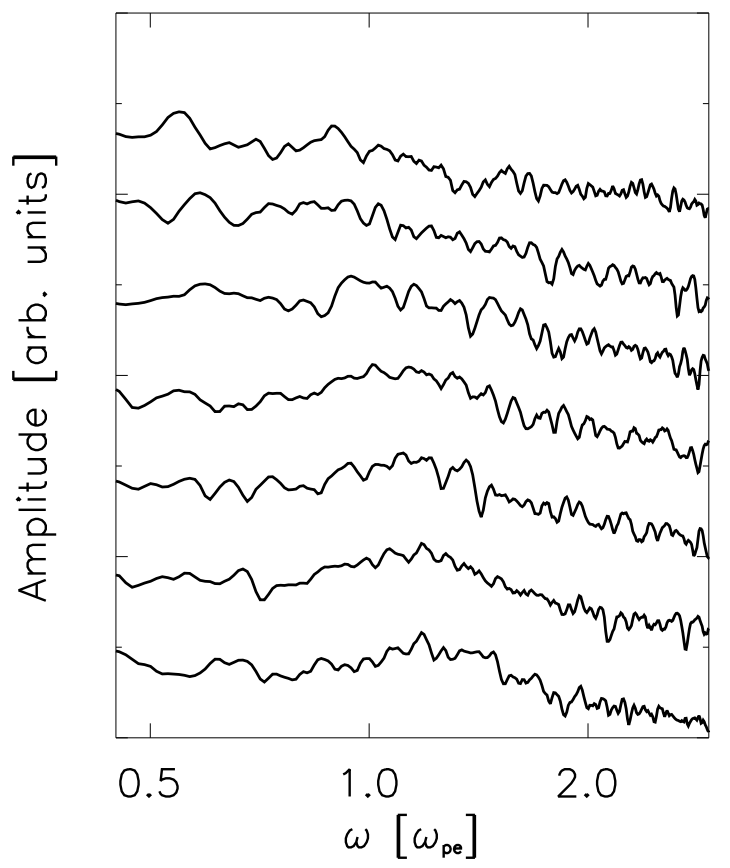

Fig. 16. Double logarithmic presentation of the high frequency spectrum of the oscillations at selected positions shown in Fig. 15 by open dots. Frequencies are normalized by the plasma frequency of the plasma at large distances from the particle, see also Fig. 14.

rest in a thermal plasma, the results are basically independent of the particle material, and corresponds to what will be found for a Langmuir probe at floating potential. Important differences between dust grains consisting of insulating and conducting materials, respectively, are found when such particles are exposed to a streaming plasma.

We have presented figures for the potential distributions, and note that for scales much larger than the Debye length, we can assume quasi-neutrality, $n_{e} \approx n_{i}$. For the relatively slow phenomena studied here, the electron component can, in addition, be considered to be locally in a Boltzmann equilibrium for the given thermal plasma, $n_{e} / n_{0} \approx \exp \left(e \phi / \kappa T_{e}\right) \approx 1+e \phi / \kappa T_{e}$, and consequently the plasma potential is representative for the large scale variation of the bulk plasma density as well, although deviations can occur on scales comparable to the Debye length.

Several results of our studies can have general interest. Thus we note that the Mach cone at supersonic flows is most clearly observed for conducting particles, see Fig. 7. The cone is present also in the case of insulating material, see Fig. 6, but for this case the potential and density variations are completely dominated by the wake, directly behind the dust grain.

By enhancing the electron-ion temperature ratio we achieve an enhancement of the ambipolar electric fields: this effect will be particularly conspicuous for an obstacle in a 
streaming plasma. For the case of an insulating material shown in Fig. 13 we note that the plasma is focused behind the dust grain, but find also that this effect can be absent for the case of a conducting material, see Fig. 15. Here the density depletion of the wake is reduced and the electric fields that build up are not as strong as for the insulating case. The focusing in Fig. 13 is manifested by the slight narrowing of the equi-density contours, in particular their inward curvature as compared to Fig. 15. The possibility of plasma focusing in this context has been discussed before (Melands $\varnothing$ and Goree, 1995; Maiorov et al., 2000; Fortov et al., 2005), but for different geometries. The focusing requires a strong ambipolar electric field pointing into the cavity region, which in turns requires a large electron pressure. In the simulations we found that the focusing effect required a relatively large temperature ratio in order to be noticeable: we consider the present case with $T_{e} / T_{i}=5$ as somewhat marginal.

A macroscopic dust particle obtains a dipole moment when it has a relative motion with respect to the plasma. Our results are summarized in Figs. 12a and b for insulating and perfectly conducting materials, respectively (Lafon et al., 1981). We analyzed also the effect of different surface conditions, again using the two dust-grain shapes illustrated in Fig. 1. There is a significant difference between conductors and insulators, the dipole moment being much larger for the latter material. The surface conditions seem not to be critical for the insulator case: the charge is predominantly accumulated on the outer surface, with only little penetrating into deep cavities. For the conductor, where charges are redistributed to cancel internal electric fields, it seems that the surface conditions are slightly more significant. The main differences between the two cases illustrated in Fig. 1 are explained by the wakes forming behind the objects. Since charges are freely moving in the conducting particles, the dipole moment is to a great extent "short-circuited" for this case. The dipole moment of the charged conducting dust particle is what is needed to neutralize the electric fields induced from the charge distribution in the surrounding plasma in order to make the internal electric field vanishing. In order to compensate an electric field pointing along the flow direction, the surface charges on the conducting material have to contribute with an electric field in the opposite direction. Consequently the direction of the dipole moment for the conducting material is opposite of the direction found for the insulator. There is no simple analytical relation between the dipole moments of insulating and conducting materials.

As evident, our results rely on a two-dimensional geometry. Most of the results remain qualitatively applicable also for a fully three-dimensional case. A comparison of the two cases has been carried out by Melands $\varnothing$ and Goree (1995), although their simulations did not use a self-consistently obtained charging of the object. The ion focusing effect will generally be more pronounced for a sphere as compared to a cylinder with same radius.
The results of the present study refer to a system which is in thermal equilibrium in the rest frame of the plasma. The case where the plasma has beam components, thus being out of thermal equilibrium, contains many parameters, and a complete study of the relevant parameter space is hardly feasible. This problem is not discussed in any further detail here, but we mention that our codes can cover such cases as well. Selected problems will be discussed later in a different context. We have several places explicitly used that typical scale sizes for the dust particles were larger than $\lambda_{D}$. Many arguments are readily generalized to cases where the opposite inequality holds. The reference floating potentials and charges then have to be derived from orbit theory. For these cases the numerical analysis has to rely on very fine grid resolutions, where the efficiency of a PIC-code is not the optimum.

Acknowledgements. We thank O. Skjæraasen and S. Børve for valuable discussions of numerical problems. Parts of this work were supported by the Research Council of Norway.

Edited by: T. Hada

Reviewed by: two anonymous referees

\section{References}

Armstrong, R. J., Liland, K. B., and Trulsen, J.: Plasma simulation of a Langmuir probe with normal magnetic-field, Physica D, 60, 160-164, 1992.

Barston, E. M.: Electrostatic oscillations in inhomogeneous cold plasmas, Ann. Phys. (NY), 29, 282-303, 1964.

Bendat, J. S. and Piersol, A. G.: Random data, Analysis and Measurement Procedures, John-Wiley \& Sons, New York, 2 edn., 1986.

Birdsall, C. K. and Langdon, A. B.: Plasma Physics via Computer Simulation, McGraw-Hill, New York, 1985.

Boeuf, J. P. and Punset, C.: Physics and modelling of dusty plasmas, in: Dusty Plasmas, edited by: Bouchoule, A., John Wiley \& Sons, 1999.

Chen, F. F.: Introduction to Plasma Physics and Controlled Fusion, vol. 1, Plenum Press, New York, 2 edn., 1984.

Eriksson, A. I. and Boström, R.: Measurements of plasma density fluctuations and electric wave fields using spherical electrostatic probes, Tech. Rep. IRF Scientific Report 220, Swedish Institute of Space Physics, 1995.

Fortov, V. E., Ivlev, A. V., Kharpak, S. A., Kharpak, A. G., and Morfill, G. E.: Complex (dusty) plasmas: Current status, open issues, perspectives, Physics Reports, 421, 1-103, 2005.

Guio, P. and Pécseli, H. L.: Radiation of sound from a charged dust particle moving at high velocity, Plasma Phys., 10, 2667-2676, 2003.

Guio, P. and Pécseli, H. L.: Phase space structures generated by an absorbing obstacle in streaming plasmas, Ann. Geophys., 23, 853-865, 2005,

http://www.ann-geophys.net/23/853/2005/.

Havnes, O., Li, F., Melandsø, F., Aslaksen, T., Hartquist, T. W., Morfill, G. E., Nitter, T., and Tsytovich, V.: Diagnostic of dusty plasma conditions by the observation of Mach cones caused by dust acoustic waves, J. Vac. Sci. Technol. A, 14, 525-528, 1996. 
Homann, A., Melzer, A., and Piel, A.: Measuring the charge on single particles by laser-excited resonances in plasma crystals, Phys. Rev. E, 59, R3835, 1999.

Huld, T., Pécseli, H. L., and Rasmussen, J. J.: Ion-acoustic wave propagation in plasma with ion beams having a finite cross section, IEEE Trans. Plasma Sci., 18, 149-158, 1990.

Hutchinson, I. H.: Ion collection by a sphere in a flowing plasma: 3. Floating potential and drag force, Plasma Phys. Contr. Fusion, 47, 71-87, 2005.

Ivlev, A. V., Morfill, G., and Fortov, V. E.: Potential of a dielectric particle in a flow of a collisionless plasma, Phys. Plasmas, 6, 1415-1420, 1999.

Kwok, D. T.-K., Oates, T. W. H., McKenzie, D. R., and Bilck, M. M. M.: Determination of the equilibrium ion sheath in the drifting plasma by numerical simulations, IEEE Trans. Plasma Sci., 31, 1044-1051, 2003.

Lafon, J.-P. J., Lamy, P. L., and Millet, J.: On the electrostatic potential and charge of cosmic grains, Astron. Astrophys., 95, 295303, 1981.

Lapenta, G.: Effect of dipole moments on the coagulation of dust particles immersed in plasmas, Physica Scripta, 57, 476-480, 1998.

Lapenta, G.: Simulation of charging and shielding of dust particles in drifting plasmas, Phys. Plasmas, 5, 1442-1447, 1999.

Laroussi, M., Mendis, D. A., and Rosenberg, M.: Plasma interaction with microbes, New J. Phys., 5, 41.1-41.10, 2003.

Lochte-Holtgreven, W. (Ed.): Plasma Diagnostics, North-Holland, Amsterdam, 1968.

Maiorov, S. A., Vladimirov, S. V., and Cramer, N. F.: Plasma kinetics around a dust grain in an ion flow, Phys. Rev. E, 63, 017401 , 2000.

Mälkki, A.: Analysis of the effect of artificial ion masses on currentdriven electrostatic instabilities, J. Geophys. Res., 99, 6045$6065,1994$.

Manweiler, J., Armstrong, T. P., and Cravens, T. E.: Complex charge distributions of dielectric dust grains due to plasma flow, J. Plasma Phys., 63, 269-283, 2000.

Matsoukas, T. and Russell, M.: Particle charging in low-pressure plasmas, J. Appl. Phys., 77, 4285-4292, 1995.

Melands $\varnothing$, F. and Goree, J.: Polarized supersonic plasma flow simulation for charged bodies such as dust particles and spacecraft, Phys. Rev. E, 52, 5312-5326, 1995.
Motley, R. W.: Q Machines, Academic Press, New York, 1975.

Nakagawa, T. and Iizima, M.: Pitch angle diffusion of electrons at the boundary of the Lunar wake, Earth, Planets and Space, 57, 885-894, 2005.

Piel, A. and Melzer, A.: Dynamical processes in complex plasmas, Plasma Phys. Control. Fusion, 44, R1-R26, 2002.

Raadu, M. A. and Shafiq, M.: Shielding of a slowly moving test charge in a dusty plasma with dynamical grain charging, Phys. Plasmas, 10, 3484-3491, 2003.

Shafiq, M. and Raadu, M. A.: Delayed shielding of a slowly moving test charge in a dusty plasma with dynamical grain charging, IEEE Trans. Plasma Sci., 32, 627-631, 2004.

Shukla, P. K. and Mamun, A. A.: Introduction to Dusty Plasmas, Institute of Physics Publishing, Bristol and Philadelphia, 2002.

Singh, N., Thiemann, H., and Schunk, R. W.: Studies of counterstreaming plasma expansion, Phys. Scripta, 33, 355-369, 1986.

Singh, N., Wright Jr., K. H., Stone, N. H., Samir, U., and Hwang, K. S.: On the interpretation of measured ion streams in the wake of the shuttle orbiter in terms of plasma expansion processes, J. Geophys. Res., 94, 12 075-12 080, 1989.

Sivukhin, D. V.: Coulomb collisions in a fully ionized plasma, in: Reviews of Plasma Physics, edited by: Leontovich, M. A., vol. 4, pp. 93-241, Consultants Bureau, New York, 1966.

Skøelv, A., Armstrong, R. J., and Trulsen, J.: Ion-beam diagnostics by means of plane Langmuir probes, Phys. Fluids, 27, 2744 2751, 1984.

Stenzel, R. L., Williams, R., Aguero, R., Kitazaki, K., Ling, A., Mcdonald, T., and Spitzer, J.: Novel directional ion energy analyzer, Rev. Scientific Instr., 53, 1027-1031, 1982.

Taccogna, F., Longo, S., and Capitelli, M.: PIC model of the ion collection by a Langmuir probe, Contrib. Plasma Phys., 44, 594600, 2004.

Teodoru, S., Tskhakaya Jr., D., Kuhn, S., Tskhakaya Sr., D. D., Schrittwieser, R., Ioniţă, C., and Popa, G.: Kinetic (PIC) simulations for a plane probe in a collisional plasma, J. Nuc. Mat., 337, 1111-1115, 2005.

Weber, W. J., Armstrong, R. J., and Trulsen, J.: Ion-beam diagnostics by means of an electron-saturated Langmuir probe, J. Appl. Phys., 50, 4545-4549, 1979.

Zhdanov, S. K., Ivlev, A. V., and Morfill, G. E.: Non-Hamiltonian dynamics of grains with spatially varying charges, Phys. Plasmas, 12, $072312,2005$. 